\title{
Efficacy of Liraglutide in clinical practice: Single centre experience
}

\author{
Muhammad Owais Rashid', Sumerah Jabeen², \\ Adeel Khoja ${ }^{3}$, Najmul Islam ${ }^{4}$
}

\begin{abstract}
Background and Objective: GLP-one receptor agonists are amongst the unique antidiabetes medications that have significant metabolic and cardiovascular benefits in addition to glucose lowering effect. To best of our knowledge, there is no published data on efficacy of liraglutide use among Pakistani population.Our objective was to ascertain the efficacy of liraglutide use among type two diabetes patients.

Methods: This retrospective study was conducted at the Endocrinology Clinics of Aga Khan University Hospital (AKUH) Karachi, Pakistan during the period from July 01, 2016 to 30th June, 2017. Liraglutide was prescribed to 68 obese type two diabetes patients with uncontrolled diabetes taking more than one oral medication \pm insulin. Starting dose of Liraglutide was $0.6 \mathrm{mg}$, which was increased to $1.2 \mathrm{mg}$ after 1-2 weeks with further increment to $1.8 \mathrm{mg} /$ day based on tolerance and individual patient preference. Dose of other diabetes medications was adjusted according to clinical judgment whereas Dipeptidyl peptidase4(DPP-4) inhibitors were discontinued.

Results: Mean age of cohort was 55 years ( $\mathrm{SD}=10.94$ years) with median body mass index of $36.45 \mathrm{~kg} / \mathrm{m}^{2}$ and majority (57.35\%) were on a dose of $1.2 \mathrm{mg}$ of Liraglutide per day. Median $\mathrm{HbA}_{1} \mathrm{c}$ reduced to $7.50 \%$ and $7.40 \%$ at three months and six months respectively vs $8.45 \%$ at baseline. Mean reduction in weight after three month was two kilograms and at six months, it was 1.38 kilograms respectively.

Conclusion: Liraglutide as add on therapy demonstrated favourable HbA1c and weight reduction in obese uncontrolled type two Diabetes Pakistani subjects.
\end{abstract}

KEYWORDS: Glucagon like peptide-1(GLP-1) agonist, Liraglutide, Type 2 diabetes.

doi: https://doi.org/10.12669/pjms.36.3.358

How to cite this:

Rashid MO, Jabeen S, Khoja A, Islam N. Efficacy of Liraglutide in clinical practice: Single centre experience. Pak J Med Sci. 2020;36(3):432-437. doi: https://doi.org/10.12669/pjms.36.3.358

This is an Open Access article distributed under the terms of the Creative Commons Attribution License (http://creativecommons.org/licenses/by/3.0), which permits unrestricted use, distribution, and reproduction in any medium, provided the original work is properly cited.

1. M. Owais Rashid MBBS, FCPS (Medicine), FCPS (Endocrinology), Consultant Endocrinologist, Section of Endocrinology,

2. Sumerah Jabeen, FCPS (Medicine).

Fellowship in Diabetes \& Endocrinology,

3. Adeel Khoja, MBBS.

Senior Instructor Research,

4. Najmul Islam, MBBS, FRCP.

Consultant Endocrinologist, Section Head Endocrinology,

1,3,4:Department of Medicine,

1-4: Aga Khan University Hospital,

Karachi, Pakistan.

Correspondence:

Muhammad Owais Rashid

Consultant Endocrinologist,

Aga Khan University Hospital,

Karachi, Pakistan.

E-mail: owais.rashid@aku.edu

* Received for Publication:

January 11, 2019

* $1^{\text {st }}$ Revision Received:

* $2^{\text {nd }}$ Revision Received:

* Final Revision Accepted:
January 14, 2020

January 20, 2020
October 18, 2019

\section{INTRODUCTION}

Type 2 diabetes being a complex metabolic disorder remains an ever-increasing global health care issue. While glycemic control is associated with reductions in the risk of micro vascular complications, the macro vascular benefits of glycemic control are not very clear. ${ }^{1}$ The reason might be that the benefits of improved glycemic control take a long time to influence the progression of atherosclerotic vascular disease.

There are multiple treatment options available for patients with diabetes, many of which are either weight neutral, cause weight gain or promote weight loss. One of the disadvantage of having these multiple treatment options is that, the wide range of options may cause problem for 
the health care provider to prescribe the optimal regimen. In making these decisions, the prescriber should consider patient's disease progression, comorbidities, and concomitant treatment. ${ }^{2}$

After decades of disappointment in attempting to control cardiovascular disease progression in type 2 diabetes with careful glycemic control,a new found hope that newer drugs, particularly the Glucagon like peptide 1 receptor (GLP-1) agonist and the sodium glucose co transporter 2 SGLT-2 inhibitors have cardiovascular benefits independent of glycemic control has led to a new dimension in the management of type 2 diabetes. ${ }^{3}$ Despite its role in glucose homeostasis, the GLP-1 receptor is amazingly widely distributed throughout the body, including heart. GLP1 may exert its effects through both receptordependent and receptor-independent mechanism and through the actions of both the intact peptide and its metabolites. ${ }^{4}$ Injectable GLP-1 receptor agonist mimics endogenous GLP-1 by stimulating pancreatic insulin secretion. They have beneficial nonglycemic metabolic effects, notably the potential for weight loss. ${ }^{5}$

Liraglutide a long acting GLP-1 receptor agonist has been approved for the treatment of type 2 diabetes. ${ }^{6}$ To assess the long-term effects of liraglutide on cardiovascular outcomes and other clinically important events, the Liraglutide Effect and Action in Diabetes: Evaluation of Cardiovascular Outcome Results (LEADER) trial was initiated in $2010 .{ }^{1}$ In the LEADER's trial, 9340 patients with type 2 diabetes at high cardiovascular risk were randomized to receive the longer-acting drug liraglutide $1.8 \mathrm{mg}$ (or maximum tolerated dose) or matching placebo once daily and followed for a mean of 3.5 years. There was a $23 \%$ statistically significant $(p$-value $=$ 0.001 for non-inferiority, $p$-value $=0.01$ for superiority) reduction in the primary end point, including a $22 \%$ reduction in cardiovascular mortality $(p$-value $=0.007)$ and $15 \%$ reduction in total mortality $(p$-value $=0.02) .{ }^{3}$ Gastrointestinal adverse effects were more common in the patients randomized to liraglutide, the trial concluded that the patients in liraglutide group had significantly reduced risk of cardiovascular events and they had better micro vascular outcomes then the group with standard therapy. ${ }^{1}$ A few studies have been conducted on South Asian population in India and all of these studies concluded that, liraglutide significantly improves glycemic control with low risk of hypoglycemia and is associated with significant weight loss in Indian patients with type 2 diabetes mellitus. ${ }^{7}$ To best of our knowledge, there is no published data on efficacy of liraglutide use among Pakistani population.

Hence, this study aimed to assess the efficacy of liraglutide in terms of glycemic control and weight reduction in type 2 diabetes patients Pakistani population during routine clinical practice at outpatient clinic.

\section{METHODS}

This retrospective study was conducted at the Endocrinology Clinics of Aga Khan University Hospital (AKUH) Karachi, Pakistan during the period from July 01, 2016 to 30th June, 2017. This study was approved by the ethical committee (Ref. No. 4911-Med-ERC-17) of the Aga Khan University.

Liraglutide was prescribed to 68 obese (BMI $>23 \mathrm{~kg} / \mathrm{m} 2$ ) type 2 diabetes patients with uncontrolled diabetes $(\mathrm{HbA} 1 \mathrm{C}>7 \%)$. All patients were on more than one oral hypoglycemic agents with or without insulin. DPP4 inhibitors were stopped if patients were on it whereas other antidiabetes medication were adjusted according to clinical judgment. All patients were advised to start $0.6 \mathrm{mg} /$ day of liraglutide. An increment in dose up to $1.2 \mathrm{mg}$ / day was done after 1-2 weeks which was later increased to $1.8 \mathrm{mg} /$ day based on tolerance and individual patient preference.

Patients aged below 16 years, non diabetics, having type 1 diabetes, not taking liraglutide were excluded from the study. Informed written consent was obtained from hospital authorities to use the data of patients. No personal detail by any means related to any patient was revealed in regard of ethical considerations.

Data of patients taking liraglutide was retrieved from hospital medical record maintained by Health Information Management System of Aga Khan University Hospital after acquiring permission. Data collection was done by means of a data collection tool specifically designed for the study, keeping in mind the indicators/ variables asked from the diabetes patient at the time of initial and follow-up visits in the diabetes clinic. A research assistant was trained to review the files of all the diabetes patients falling under the aforementioned timelines by means of a data collection tool. Data collection tool mainly covered information on demographic details of 
Graphical Representation of all

Continuous Variables in the Dataset.

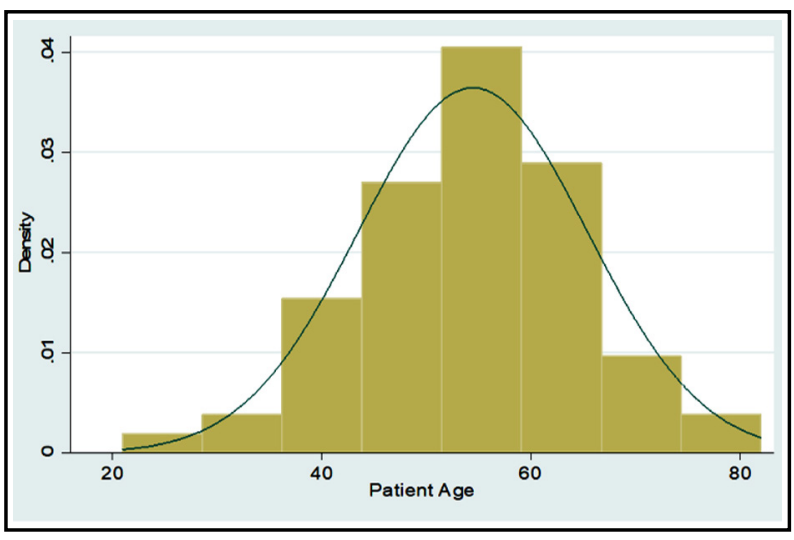

Histograms: AGE Distribution

Check: Symmetrically Distributed.

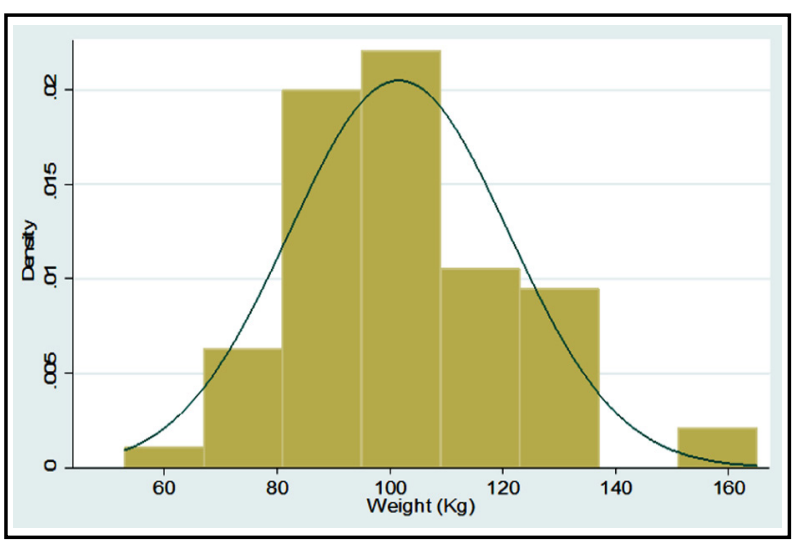

WEIGHT: Distribution Check: Symmetrically Distributed.

the patient, duration of diabetes and patients with history of Hypertension (taking anti hypertensive medication or $\mathrm{BP}>140 / 90 \mathrm{mmh}$ on home BP monitoring), Dyslipidemia (serum LDL $>100 \mathrm{mg} /$ $\mathrm{dl}$, triglycerides $>150 \mathrm{mg} / \mathrm{dl}$ ), Coronary artery disease including history of unstable angina, previous history of Myocardial infarction or coronary angioplasty, retinopathy (both proliferative and non proliferative) diagnosed and treated by ophthalmologist, neuropathy (diagnosed on previous visits with compromised vibration and pin prick sensation assessed with a calibrated tuning fork and $10 \mathrm{gm}$ monofilament testing) and nephropathy (confirmed by presence of albuminuria in urine on 2 or more occasions), medication details, changes in glycosylated haemoglobin(HbA1C) and weight, any reported side effects of liraglutide and history of discontinuation if any.

Plan of Analysis: Frequencies with percentages were reported for categorical variables for e.g.
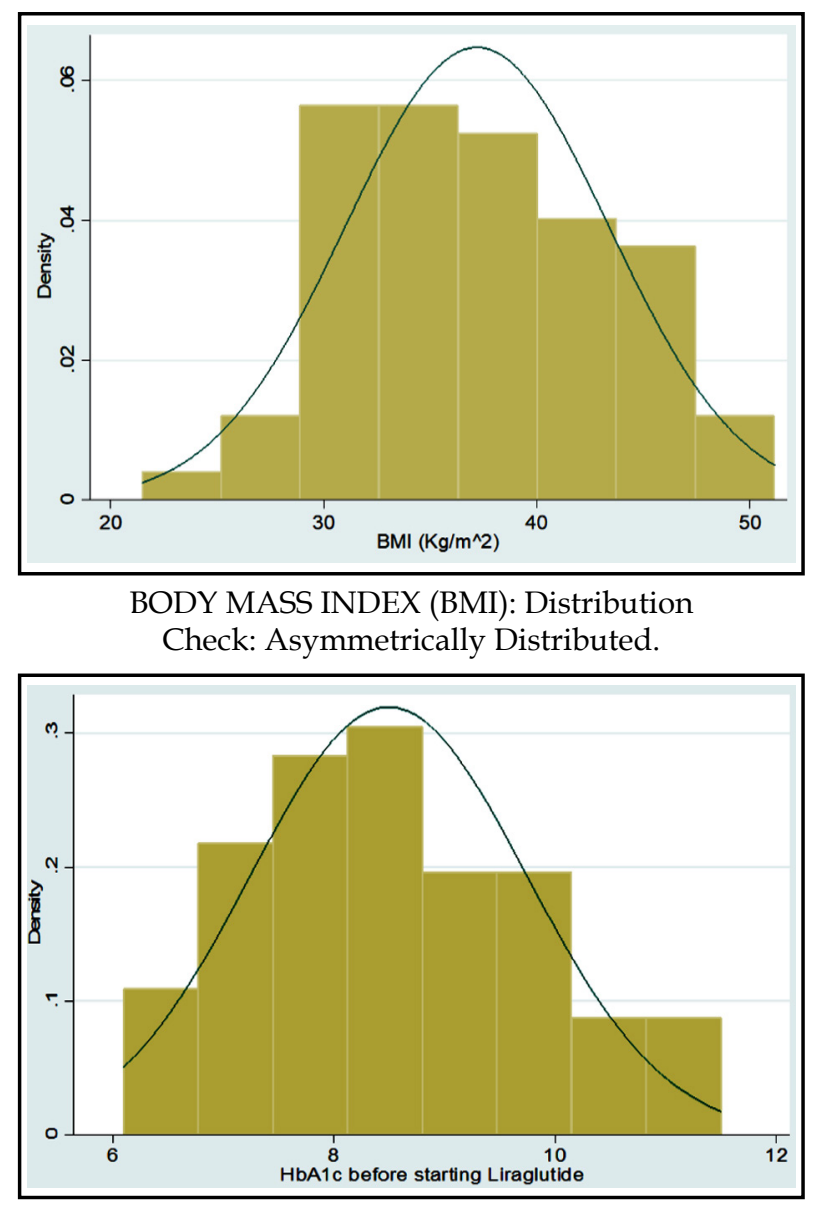

HBA1C BEFORE STARTING LIRAGLUTIDE:

Distribution Check: Asymmetrical Distribution.

gender, associated comorbids, and associated Diabetes complications, medication history before adding liraglutide, side effects of Liraglutide etc. Mean with standard deviation was reported for all symmetrically distributed continuous variables while median with inter quartile range was reported for all asymmetrically distributed variables for e.g. age, duration of comorbids, baseline anthropometric measurements such as height, weight, BMI, BP, Pulse, HbA1C and subsequent followup measurements at three and six months, etc. All the analysis was performed on STATA version 12 .

\section{RESULTS}

Of the total 68 patients studied, mean age of cohort was 55 years ( $\mathrm{SD}=10.94$ years). Among them $50 \%$ were male, having a median diabetes duration of 13 years. More than four-fifth of our study population had dyslipidemia and hypertension and less than $15 \%$ had coronary 
Table-I: Descriptive analysis of patients on liraglutide.

\begin{tabular}{|c|c|c|}
\hline \multicolumn{2}{|c|}{$\begin{array}{l}\text { S. Variables } \\
\text { No. }\end{array}$} & $n=68(\%)$ \\
\hline 1 & Age* $^{*}$ & $54.47 \pm 10.94$ \\
\hline 2 & Gender & \\
\hline & (a) Male & $34(50 \%)$ \\
\hline & (b) Female & $34(50 \%)$ \\
\hline 3 & Duration of Diabetes* & $13(7-19)$ \\
\hline 4 & Presence of Dyslipidemia & $57(83.82 \%)$ \\
\hline 5 & Presence of Hypertension & $61(89.71 \%)$ \\
\hline 6 & $\begin{array}{l}\text { Presence of Coronary } \\
\text { Artery Disease }\end{array}$ & $9(13.24 \%)$ \\
\hline 7 & Presence of Retinopathy & $4(5.90 \%)$ \\
\hline 8 & Presence of Neuropathy & $5(7.35 \%)$ \\
\hline 9 & Presence of Nephropathy & $3(4.41 \%)$ \\
\hline 10 & Body Mass Index $\left(\mathrm{kg} / \mathrm{m}^{2}\right)^{*}$ & $36.45(32.40-41.9)$ \\
\hline 11 & Dose of Liraglutide (mg) & \\
\hline & (a) 0.6 & $7(10.29 \%)$ \\
\hline & (b) 1.2 & $39(57.35 \%)$ \\
\hline & (c) 1.8 & $22(32.35 \%)$ \\
\hline 12 & $\begin{array}{l}\text { Participants on Sulfonylurea } \\
\text { before liraglutide }\end{array}$ & $30(44.12 \%)$ \\
\hline 13 & $\begin{array}{l}\text { Participants on Metformin } \\
\text { before liraglutide }\end{array}$ & $64(94.12 \%)$ \\
\hline 14 & $\begin{array}{l}\text { Participants on Thiazolidinedione } \\
\text { before liraglutide }\end{array}$ & $4(5.88 \%)$ \\
\hline 15 & $\begin{array}{l}\text { Participants on DDP-4 inhibitors } \\
\text { before liraglutide }\end{array}$ & $33(48.53 \%)$ \\
\hline 16 & $\begin{array}{l}\text { Participants on Basal Insulin } \\
\text { before liraglutide }\end{array}$ & $22(30.88 \%)$ \\
\hline 17 & $\begin{array}{l}\text { Participants on Pre-Mixed Insulin } \\
\text { before liraglutide }\end{array}$ & $19(27.94 \%)$ \\
\hline 18 & $\begin{array}{l}\text { Participants on Basal Bolus } \\
\text { Regimen before liraglutide }\end{array}$ & $22(32.35 \%)$ \\
\hline 19 & Creatinine levels of participants * & $0.8(0.7-0.95)$ \\
\hline
\end{tabular}

artery disease (Table-I). Median body mass index of our study participants was $36.45 \mathrm{~kg} / \mathrm{m}^{2}$, and majority of them $(57.35 \%)$ were on a dose of 1.2 mg of Liraglutide per day. Study participants were mostly on maximum doses of sulfonylureas (44\%), metformin (94\%), DPP-4 inhibitors (49\%), basal insulin $(31 \%)$, pre-mixed insulin $(28 \%)$, basal bolus regimen (32\%) either alone or in combination before addition of Liraglutide to the treatment regimen. DPP-4 inhibitors were discontinued in all patients once liraglutide was introduced and dosage of other antidiabetes medications adjusted. Median $\mathrm{HbA}_{1} \mathrm{c}$ at baseline was $8.45 \%$ and it reduced to $7.50 \%$ and $7.40 \%$ at three months and six months post-Liraglutide respectively. The mean reduction in weight after three months of Liraglutide was 2 kilograms and at six months, it was 1.38 kilograms respectively. There was no significant difference noted in the systolic and diastolic blood pressures and the pulse rate at three and six months from the baseline, respectively (Table-I).

Side-effects reported after the start of Liraglutide were mainly nausea $(9 \%)$, vomiting $(6 \%)$, diarrhoea $(7 \%)$ and others $(12 \%)$ which included headache, dizziness and constipation. In addition to this, Liraglutide was discontinued due to the side effects and cost in about seven participants $(10 \%)$ of our study cohort and therefore they were not included in the final analysis.

\section{DISCUSSION}

This small scale study verifies that Liraglutide as an add on therapy was beneficial for both HbA1c and weight reduction in Pakistani subjects with Diabetes Mellitus (T2DM). The HbA1c reduction at 3 months from baseline in our study was $0.95 \%$ and $1.05 \%$ at 6 months. There was $-2.00 \pm 3.10 \mathrm{~kg}$ weight reduction at three months and $-1.38 \pm 3.27$ $\mathrm{kg}$ at 6 months. The $\mathrm{HbA1c}$ reduction in our study corresponds to the maximum $\mathrm{HbA1c}$ reduction noted in LEAD -4 trial which showed $1.5 \%$ reduction at 1.2 and $1.8 \mathrm{mg}$ of Liraglutide doses at 24 weeks of treatment. ${ }^{8}$ Although the mean weight reduction in our study noted at six months of treatment was less as anticipated but it was comparable to weight reduction in LEAD trials which was also less than $3 \mathrm{~kg}$ with the exception of LEAD-6 which showed a weight reduction of $3.24 \mathrm{~kg} .{ }^{9}$ The probable explanation to this could be that weight loss demonstrated with Liraglutide is dose dependant whereas in our study more than $50 \%$ subjects were on $1.2 \mathrm{mg}$ doses. ${ }^{10-12}$ No change in systolic, diastolic blood pressures and pulse at baselines and at six months post treatment from the baseline was noted although this was not the primary objective and includes several confounding factors like retrospective design, medication adherence, operator variability etc. Gastrointestinal side effects were the commonly reported adverse effects among the study participants and seven participants withdrew liraglutide owing to these side effects. Financial constraints remained an important factor in continuing patients on Liraglutide therapy.

Following the LEADER trial which showed significant reduction in the composite outcome of the first occurrence of death from CV causes, 
Table-II: Comparison of variables from baseline following Liraglutide treatment.

\begin{tabular}{|c|c|c|c|c|}
\hline $\begin{array}{l}\text { S. } \\
\text { No. }\end{array}$ & Variables & At Baseline & At 3 months & At 6 months \\
\hline 1 & HbA1c (at baseline) of participant (\%)* & $8.45(7.55-9.45)$ & $7.50(6.70-8.6)$ & $7.40(6.70-7.90)$ \\
\hline 2 & $\begin{array}{l}\text { Change in weight after three months } \\
\text { post-Liraglutide of participants* }\end{array}$ & - & $-2.00 \pm 3.10$ & $-1.38 \pm 3.27$ \\
\hline 3 & Systolic Blood Pressure (at baseline) of participants $(\mathrm{mmHg})^{*}$ & $138.28 \pm 17.11$ & $134.95 \pm 23.60$ & $138.07 \pm 15.60$ \\
\hline 4 & Diastolic Blood Pressure (at baseline) of participants $(\mathrm{mmHg})^{*}$ & $74.42 \pm 10.05$ & $72.93 \pm 8.80$ & $72.64 \pm 11.70$ \\
\hline 5 & Pulse Rate (at baseline) of participants (beats/min)* & $89.34 \pm 13.50$ & $90.40 \pm 14.85$ & $92.14 \pm 16.61$ \\
\hline 6 & $\begin{array}{l}\text { Side Effects reported by participants post-Liraglutide } \$ \text { : } \\
\text { 1. Nausea } \\
\text { 2. Vomiting } \\
\text { 3. Diarrhea } \\
\text { 4. Others }\end{array}$ & & $\begin{array}{l}6(8.82 \%) \\
4(5.88 \%) \\
5(7.35 \%) \\
8(11.76 \%)\end{array}$ & \\
\hline 7 & Liraglutide discontinued due to side effects \& cost & & $7(10.29 \%)$ & \\
\hline
\end{tabular}

Legends used in Table-I \& II: * All the continuous variables in the table above have been reported as either mean with standard deviation (Mean \pm SD), if they are symmetrically distributed or as median with interquartile range (Median (25th percentile -75 th percentile) if they are asymmetrically distributed.

\$ It was a multiple response question and the sum of the percentages will not be equal to 100.

non-fatal myocardial infarction or nonfatal stroke in T2DM subjects with high CV risk, Liraglutide along with other GLP 1 agonists is now considered as second line therapy in high risk $\mathrm{CV}$ patients. The EASD and the ADA updated 2018 consensus statement for DM management in patients with T2 DM encourages use of GLP 1 receptor agonist and SGLT2 inhibitor for patients with clinical cardiovascular disease as an add on therapy after metformin. ${ }^{13}$ The exact mechanism influencing the beneficial effects of Liraglutide on cardiovascular benefit and weight reduction remains unknown but one probable hypotheses is reduction in visceral fat, hepatic steatosis and systemic inflammation which are considered strong contributors for cardiovascular morbidity in patients with diabetes. ${ }^{14,15}$ Bouchi et al. demonstrated the Liraglutide efficacy in reducing visceral fat and thus ameliorating hepatic steatosis, albuminuria and microinflammation in T2DM patients along with improved quality of life following treatment. ${ }^{16}$

This single centre retrospective study is the first Pakistani study in evaluating the liraglutide efficacy in T2 Diabetes Pakistani subjects. The mean duration of diabetes in our study population was 13 years but nevertheless there was a mean reduction in $\mathrm{HbA1c}$ at both three months and six months. Kaur et al. in their Liraglutide trial highlighted that weight reduction and diabetes control was irrespective of the duration of diabetes and baseline $\mathrm{HbA1c}$ thus concluding that liraglutide effectiveness could be achieved in patients with long standing diabetes as well. ${ }^{17}$ Although favourable $\mathrm{HbA1C}$ and weight reduction was found at six months post liraglutide several confounding factors like diet and physical activity could not be controlled due to retrospective design of study.

Despite the effective $\mathrm{HbA1}$ c and weight reduction Liraglutide remains an expensive treatment option for a country like Pakistan where majority of the patients do not have medical insurance. Since this is a single centre experience, prospective studies are required evaluating multiple beneficial effects of liraglutide in patients with $\mathrm{CV}$ risks and baseline albuminuria thus evaluating the new cardio protective and renal protective role of this drug.

\section{CONCLUSION}

Liraglutide as add on therapy demonstrated favourable $\mathrm{HbA1c}$ and weight reduction in a real life clinical setting of Pakistani Type-2 diabetes patients. Despite the efficacy, financial constraints remain an important set back in utilizing the maximum benefitting effects of this drug.

Acknowledgement: We would like to acknowledge Daniyaal Nadeem AKUH medical student in helping in data collection and data analysis.

\section{Source of funding: None.}

Conflict of interest: None. 


\section{REFERENCES}

1. Marso SP, Daniels GH, Brown-Frandsen K, Kristensen P, Mann JFE, Nauck MA, et al. Liraglutide and Cardiovascular Outcomes in Type 2 Diabetes. N Engl J Med. 2016;375(4):311322. doi: $10.1056 /$ NEJMoa1603827

2. Nathan DM. Finding new treatments for diabetes--how many, how fast... how good? N Engl J Med. 2007;356(5):437440. doi: 10.1056/NEJMp068294

3. Thompson PL, Davis TM. Cardiovascular Effects of Glucose-Lowering Therapies for Type 2 Diabetes: New Drugs in Perspective. Clin Therap. 2017;39(5):1012-1025. doi: 10.1016/j.clinthera.2016.10.008

4. Advani A, Bugyei-Twum A, Connelly KA. Cardiovascular Effects of Incretins in Diabetes. Canadian J Diabetes. 2013;37(5):309-314. doi: 10.1016/j.jcjd.2013.06.010

5. Ryan D, Acosta A. GLP-1 receptor agonists: Nonglycemic clinical effects in weight loss and beyond. Obesity. 2015;23(6):1119-1129. doi: 10.1002/oby.21107

6. Doggrell SA. Liraglutide, a GLP-1 receptor agonist, prevents cardiovascular outcomes in patients with type 2 diabetes. Evidence-Based Med. 2017;22(1):28. doi: 10.1136/ ebmed-2016-110566

7. Kaur P, Mishra SK, Mithal A, Saxena M, Makkar A, Sharma P. Clinical experience with Liraglutide in 196 patients with type 2 diabetes from a tertiary care center in India. Indian J Endocrinol Metab. 2014;18(1):77-82.

8. Zinman B, Gerich J, Buse JB, Lewin A, Schwartz S, Raskin P, et al. LEAD-4 Study Investigators. Efficacy and safety of the human glucagon-like peptide-1 analogliraglutide in combination with metformin and thiazolidinedione in patients with type 2 diabetes (LEAD-4 Met+ TZD). Diabetes Care. 2009;32(7):12241230. doi: $10.2337 /$ dc08-2124

9. Buse JB, Rosenstock J, Sesti G, Schmidt WE, Montanya E, Brett JH, et al. LEAD-6 Study Group. Liraglutide once a day versus exenatide twice a day for type 2 diabetes: a $26-$ week randomised, parallel-group, multinational, openlabel trial (LEAD-6). Lancet. 2009;374(9683):39-47. doi: 10.1016/S0140-6736(09)60659-0

10. Nauck M, Frid A, Hermansen K, Shah NS, Tankova $\mathrm{T}$, Mitha $\mathrm{IH}$, et al. Efficacy and safety comparison of liraglutide, glimepiride, and placebo, all in combination with metformin, in type 2 diabetes: the LEAD (liraglutide effect and action in diabetes)-2 study. Diabetes Care. 2009;32(1):84-90. doi: 10.2337/dc08-1355

11. Russell-Jones D, Vaag A, Schmitz O, Sethi BK, Lalic N, Antic $S$, et al. Liraglutidevs insulin glargine and placebo in combination with metformin and sulfonylurea therapy in type 2 diabetes mellitus (LEAD-5 met+ SU): A randomised controlled trial. Diabetologia. 2009;52(10):2046-2055. doi: 10.1007/s00125-009-1472-y
12. Garber A, Henry R, Ratner R, Garcia-Hernandez PA, Rodriguez-Pattzi H, Olvera-Alvarez, et al. LEAD-3 (Mono) Study Group. Liraglutide versus glimepiride monotherapy for type 2 diabetes (LEAD-3 Mono): a randomised, 52-week, phase III, double-blind, parallel-treatment trial. Lancet. 2009;373(9662):473-481. doi: 10.1016/S0140-6736(08)61246-5

13. Davies MJ, D' Alessio DA, Fradkin J, Kernan WN, Mathieu C, Mingrone G, et al. Management of hyperglycaemia in type 2 diabetes, 2018. A consensus report by the American Diabetes Association (ADA) and the European Association for the Study of Diabetes (EASD). Diabetologia. 2018;61(12):24612498. doi: 10.1007/s00125-019-4845-x

14. Targher G, Arcaro G. Non-alcoholic fatty liver disease and increased risk of cardiovascular disease. Atherosclerosis. 2007;191(2):235-240. doi: 10.1016/j. atherosclerosis.2006.08.021

15. Kengne AP, Batty GD, Hamer M, Stamatakis E, Czernichow S. Association of C-reactive protein with cardiovascular disease mortality according to diabetes status: Pooled analyses of 25,979 participants from four UK prospective cohort studies. Diabetes Care. 2012;35(2):396-403. doi: 10.2337/dc11-1588

16. Bouchi R, Nakano Y, Fukuda T, Takeuchi T, Murakami M, Minami I, et al. Reduction of visceral fat by liraglutide is associated with ameliorations of hepatic steatosis, albuminuria, and micro-inflammation in type 2 diabetic patients with insulin treatment: A randomized control trial. Endocr J. 2017;64(3):269-281. doi: 10.1507/endocrj.EJ16-0449

17. Kaur P, Mahendru S, Mithal A. Long-term efficacy of liraglutide in Indian patients with Type 2 diabetes in a realworld setting. Indian J Endocrinol Metab. 2016;20(5):595599. doi: $10.4103 / 2230-8210.183825$

\section{Authors' Contribution:}

MO conceived the study.

AK helped in data analysis.

MO and SJ were involved in drafting of manuscript and is responsible for integrity of research.

NI did review of manuscript. All authors read and approved the final manuscript. 